\title{
Science in Underground Laboratories and DULIA-Bio
}

\author{
Aldo lanni * \\ LNGS-INFN, L'Aquila, Italy
}

This paper reports a brief introduction to Deep Underground Laboratories (DULs) and the connection they have with research on biology in extreme environments and the effect of radiation in life. Presently, there are 14 DULs in operation worldwide. Although the main research activity in these infrastructures concerns the search for rare events in astroparticle physics and neutrino physics, DULs offer a unique opportunity to undertake experiments in astrobiology and biology in extreme environments. This is the main motivation of Deep Underground Laboratory Integrated Activity in biology (DULIA-bio) 2019 Workshop, which was held at the Gran Sasso (Italy) underground laboratory. This paper aims to give an introduction to the subject of the Workshop by reviewing the main features of DULs.

Keywords: underground laboratories, low radioactivity environment, underground biology, DULIA-bio, LNGS

\section{INTRODUCTION: DEEP UNDERGROUND LABORATORIES}

\section{OPEN ACCESS}

Edited by:

Geoffrey Battle Smith,

New Mexico State University, United States

Reviewed by: Kate Scholberg, Duke University, United States Annarita Margiotta, University of Bologna, Italy

*Correspondence:

Aldo lanni aldo.ianni@Ings.infn.it

Specialty section: This article was submitted to High-Energy and Astroparticle

Physics,

a section of the journal Frontiers in Physics

Received: 21 October 2020 Accepted: 04 January 2021 Published: 23 February 2021

Citation: lanni $A$ (2021) Science in Underground Laboratories and DULIA-Bio. Front. Phys. 9:612417. doi: 10.3389/fphy.2021.612417
Deep Underground Laboratories (DULs) are research infrastructures built under a rock overburden greater than about $1,000 \mathrm{~m}$ of water equivalent (m.w.e). The larger the overburden the greater the reduction of the muon flux from primary cosmic rays. This is a key feature for DULs. A reduced muon flux by a factor larger than $10^{5}$ allows the probing of rare events such as low energy $(\sim \mathrm{MeV})$ neutrino interactions, neutrinoless double beta decay, and dark matter particle interactions. At present, 14 DULs are in operation and three underway in a time scale of 1-7 years (the ANDES is foreseen in 2027). In Figure 1 we show the distribution of DULs around the world, updated from [1]. We notice that at present there is no DUL in operation in the Southern hemisphere. A DUL in the Southern hemisphere is of great relevance due to the atmospheric muon flux dependence on the season. The seasonal effect depends on the temperature of the atmosphere. As a matter of fact, a positive correlation between the temperature and the muon flux has been measured in several DULs. Therefore, in summer time the atmospheric muon flux on surface is larger. This effect could induce a seasonal dependence of the atmospheric muon-induced backgrounds in DULs. A comprehensive study of the atmospheric muon flux and temperature correlation has been carried out by the LVD collaboration from data taken from 1994 to 2017 [2]. The comparison of measurements of the seasonal effect induced by atmospheric muons in DULs located in the Northern and Southern hemisphere is crucial, as an example, in the evaluation of the expected annual modulation of dark matter particle interaction rate, which is a model-independent signature of dark matter. In fact, the motion of the Earth in the galactic halo produces an annual dependence of the rate with a maximum in early June. This dependence is expected to not change from one hemisphere to the other. On the contrary, any muon related background will change moving from one hemisphere to the other. Therefore, a comparison of measurements from detectors deployed in DULs in the Northern and Southern hemispheres will disentangle physics signals against backgrounds.

The muon flux in DULs as a function of the depth is shown in Figure 2. The solid line shows the muon flux for a flat surface. For some of the DULs the mountain profile affects significantly the muon flux underground, which has a characteristic angular distribution in these cases. This effect is shown 


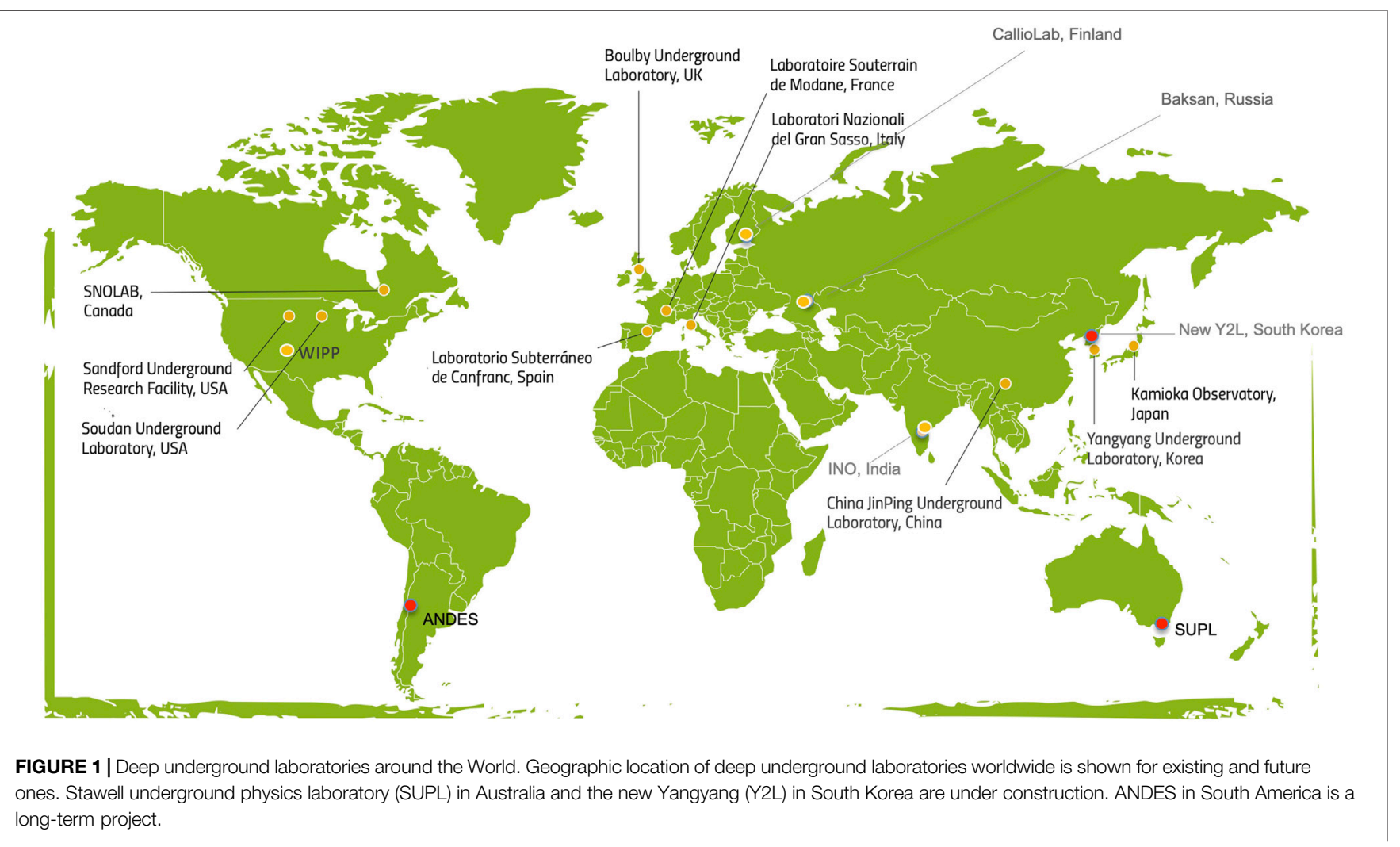

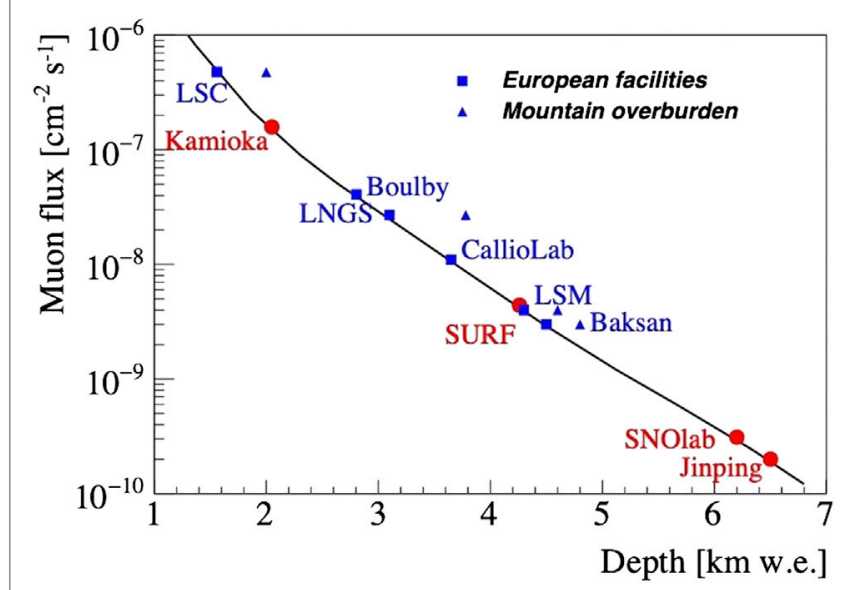

FIGURE 2 | Muon flux in underground laboratories. The muon flux in DULS is given against depth. The solid line shows the muon flux under a flat surface profile. Triangles show the maximum depth taking into account the real mountain profile.

for the Laboratorio Subterraneo de Canfranc (LSC), the Gran Sasso Laboratory (LNGS), Modane Underground Laboratory (LSM), and Baksan in the same figure.

Important and unique facilities are installed in DULs to support experimental activities. Of particular interest are the low background installations, which are mainly made by high purity germanium detectors for gamma spectroscopy. They are used for radiopurity assay of materials to be used in experimental projects. In addition, the development of experimental activities in DULs in the last 30 years has brought a significant boost in the search for rare events.

\section{Science in DULs not Related to Biology}

The main research activity in DULs concerns the search for rare events such as solar neutrino interactions, neutrinoless double beta decay, and dark matter particle interactions. In addition, nuclear interactions at low energy ( $\mathrm{keV}$ scale) between light nuclei are studied underground due to the very low cross sections. These nuclear interactions are relevant to understand energy production processes taking place inside stars [3]. Neutrinos produced by beta radioactive decays from $\mathrm{U}$ and $\mathrm{Th}$ in the interior of the Earth, so-called geo-neutrinos, are also detected in DULs [4, 5].

The scientific effort made in DULs has been awarded with two Nobel prizes in Physics in 2002 and 2015, respectively. In particular, in 2002 for the observation of neutrinos from the Sun [6, 7] and in 2015 for the discovery of neutrino oscillations $[8,9]$.

The Sun is a huge source of electron neutrinos in the $\mathrm{MeV}$ energy range. These neutrinos carry information directly from the core of the star. Massive (100-1,000 ton) water Cherenkov [8, 10] or scintillator [11] detectors with extreme radio-purity are 
needed to detect solar neutrinos. Solar neutrinos in DULs have been studied for 50 years. From this experimental activity we have learnt about neutrino oscillations, neutrino propagation in matter, and about the inner properties of energy production inside stars [12].

The neutrino can be its own antiparticle, the so-called Majorana neutrino. This property could offer an explanation for the matter-antimatter asymmetry in the Universe. Only eleven nuclei can undergo a double beta decay: two electrons and two neutrinos are emitted during the decay on the contrary of a standard beta decay, where only one electron and one neutrino are emitted. Yet, if the neutrino is a Majorana particle no neutrinos will be emitted. These nuclei offer a unique opportunity to understand whether a neutrino is a Majorana particle. A number of experiments are underway to search for a neutrinoless double beta decay. Presently, the sensitivity to this extreme rare event is of the order of $10^{26}$ years [13]. These experiments require an environment where the natural radioactivity level and the cosmic ray background are extremely low.

There are strong arguments to believe that our galaxy, as much as other galaxies, contains a dominant amount of dark matter [14]. Dark matter has been observed only through gravitational effects, such as the rotation curves of stars moving in the outer region of galaxies. Yet, in DULs it is possible to directly observe interactions of dark matter particles. This opportunity would be crucial to understand what dark matter is made of.

Besides these main research topics, in recent years DULs have become multidisciplinary infrastructures. The underground environment in DULs allows for high sensitivity studies in teleseismic and other rare phenomena, such as solid Earth tides and water effects deep below surface. The LSC, as an example, is equipped with two $70 \mathrm{~m}$-long laser interferometers [15] and a network of seismometers to characterize Newtonian noise underground.

Finally and recently, in Kamioka (Japan) an experiment to search for gravitational waves (KAGRA) has been installed in an underground environment due to the reduced background noise with respect to ground-based detectors [16].

\section{Biology in DULs and DULIA-Bio}

Life on Earth extends into deep subsurface and extreme environments, where bacteria and archaea tend to be the main living organisms. Subterranean microorganisms have been described in some detail, but most reports refer to samples taken centimeters to few meters below the surface. The literature describing microorganism inhabiting the very inside of rocks are still scarce and show a rather high diversity of microbial taxa and metabolic pathways [17]. Repeated and/ or long-term sampling substantially simplifies in the neighborhood or inside of a DUL. On the contrary, the majority of data describing the influence of deep underground conditions on life have been derived from experiments carried out in DULs. In particular, the exploration of changes in living organisms exposed to low radioactivity has been challenging the initial belief of a decrease in biological risk. Multiple experiments with a variety of methods and organisms sampled on surface and in DULs have demonstrated a significant negative impact on life due to the lack of radiation. However, for a conclusive understanding more studies are needed. Therefore, DULs offer an opportunity to advance in these scientific quests in a multidisciplinary environment, helped by experienced physicists that have been working in low background characterization for several decades. In this spirit, the first DULIA-bio conference was organized by the Canfranc Underground Laboratory (Spain) in 2015 (https://indico. cern.ch/event/436589/). In the second meeting, hosted by the Underground Laboratory at Gran Sasso (Italy) in 2019 (https://agenda.infn.it/event/19116/), new experiments, installed in underground laboratories at Sudbury, Modane, Canfranc, Boulby, and Sichuan, showed an increased research effort in this framework with respect to the pioneering works done at LNGS and WIPP $[18,19]$. The Boulby laboratory in the United Kingdom agreed to host the next DULIA-bio conference, likely in 2021.

\section{DISCUSSION}

DULs are large and unique infrastructures equipped with a number of ancillary facilities to support research activity. For years the main topic of research in DULs has been focused on astroparticle physics (dark matter, nuclear astrophysics, neutrino astrophysics) and neutrino physics (neutrinoless double beta decay and neutrino oscillations). However, in recent years DULs are diversifying the research activities to include geophysics and biology. As it has been mentioned above, DULs offer a unique opportunity to support research on astrobiology, biology in extreme environments, and radiation effects on life. The above discussed Workshops, named DULIA-bio, offered a forum where progress achieved and major needs to go further in this framework could be identified and addressed. Among current requirements, there is a need for consistent methods and conditions to improve the control on all the environmental conditions in order to reduce systematic uncertainties. In particular, materials where biological samples are stored underground should be well characterized, placed inside lead and polyethylene shields with radon free air atmosphere, so that an accurate background model can determine the nature and levels of radioactivity and also, very importantly, the energy spectra of the different sources. The impact of low radioactivity on life may well be diverse. In addition, standardization among laboratories is needed, and toward this goal the model organism Drosophila is being discussed as a common model organism. Also, standard methods would make results more comparable in different laboratories. In conclusion, these improvements and synergy with DULs may well allow this community to further understand the impact of natural radioactivity in life, which addresses the deeper question of the influence of randomness in life. We believe that evolution at some level involves randomness. The rate of interaction of 
an organism with external factors, such as ionizing radiation, as an example, could be a source of randomness. Due to a substantial reduction in muon-induced ionization and muoninduced radioactivity deep underground, DULs, by comparison with exposure on surface, offer a unique opportunity to study how, at least, this factor plays a role in the concept of random processes, which might affect the reproduction of cells and the development of organisms. The role which could be played by DULs is crucial. Therefore, we aim for future DULIA-bio meetings to reinforce synergies between DULs and the community involved in this specific research topic.

\section{REFERENCES}

1. Ianni A. Review of technical features in underground laboratories. Int J Mod Phys A (2017) 32:1743001. doi:10.1142/S0217751X17430011

2. Agafonova NY, Aglietta M, Antonioli P, Ashikhmin VV, Bari G, Bruno, et al. The LVD collaboration, characterization of the varying flux of atmospheric muons measured with the large volume detector for 24 years. Phys Rev D (2019) 100:062002. doi:10.1103/PhysRevD.100. 062002

3. Formicola A, Imbriani G. Introduction to experimental nuclear astrophysics. Eur Phys J Plus (2019) 134(3):89. doi:10.1140/epjp/i2019-12497-1

4. Gando A, Gando Y, Hanakago H, Ikeda H, Inoue K, Ishidoshiro, et al. Reactor on-off antineutrino measurments with KamLAND. Phys Rev D (2013) 88: 033001. doi:10.1103/PhysRevD.88.033001

5. Agostini M, Altenmuller K, Appel S, Atroshchenko V, Bagdasarian Z, Basilico $\mathrm{D}$, et al. Comprehensive geoneutrino analysis with Borexino. Phys Rev D (2020) 101:012009. doi:10.1103/PhysRevD.101.012009

6. Cleveland BT, Daily T, Davis R, Distel JR, Lande K, Lee CK, et al. Measurement of the solar electron neutrino flux with the Homestake chlorine experiment. Astrophys J (1998) 496:505-26. doi:10.1086/305343

7. Hirata KS, Kajita T, Kifune T, Kihara K, NakahataNakamura M, et al. Observation of $8 \mathrm{~B}$ solar neutrinos in the Kamiokande-II detector. Phys Rev Lett (1989) 63:16-9. doi:10.1103/PhysRevLett.63.16

8. Abe K, Haga Y, Hayato Y, Ikeda M, Iyogi K, Kameda J, et al. The superkamiokande collaboration, Solar neutrino measurements in superkamiokande IV. Phys Rev D (2016) 94:052010. doi:10.1103/PhysRevD.94. 052010

9. Aharmim B, Ahmed SN, Anthony AE, Barros N, Beier EW, Bellerive A, et al. The SNO Collaboration, combined analysis of all three phases of solar neutrino data from the sudbury neutrino observatory. Phys Rev C (2013) 88:025501. doi:10.1103/PhysRevC.88.025501

10. Aharmim B, Ahmed SN, Anthony AE, Barros N, Beier EW, Bellerive A, et al. The SNO collaboration. Nucl Instrum Methods (2000) 449:172. doi:10.1016/ S0168-9002(99)01469-2

\section{AUTHOR CONTRIBUTIONS}

The author confirms being the sole contributor of this work and has approved it for publication.

\section{ACKNOWLEDGMENTS}

The author would like to thank LNGS and all technical Services for the support in the organization of the DULIA-bio 2019 Workshop. The author would like to thank Carlos Peña-Garay for comments and suggestions.

11. Agostini M, Altenmuller K, Appel S, Atroshchenko V, Bagdasarian Z, Basilico D, et al. The borexino collaboration. Astropart Phys (2002) 16:205-34. doi:10. 1016/S0927-6505(01)00179-7

12. Oberauer L, Ianni A, Serenelli A. Solar neutrino physics. Berlin: Wiley VCH (2020). $240 \mathrm{p}$.

13. Kim Y-H. Neutrinoless double beta decay experiment. Available from: arXiv: 2004.02510v1 (Accessed April 6, 2020).

14. Profumo S, Giani L, Piattella OF An introduction to dark matter particles. Universe (2019) 5:213. doi:10.3390/universe5100213

15. Amoruso A, Crescentini L Free core resonant parameters from diurnal strain tides recorded by the Gran Sasso (Italy) and Canfranc (Spain) underground geodetic interferometers. Geophys J Int (2020) 198:923-36. doi:10.1111/j.1365246X.2012.05440.x

16. Akutsu T, Ando M, Arai K, Arai Y, Araki S, Araya A, et al. KAGRA Collaboration, KAGRA: 2.5 generation interferometric gravitational wave detector. Nature Astron (2019) 3(1):35-40. doi:10.1038/s41550-018-0658-y

17. Wilkins M, Daly RA, Mouser PJ, Trexler R, Sharma S, Cole DR, et al. Trends and future challenges in sampling the deep terrestrial biosphere. Front Microbiol (2014) 5:481. doi:10.3389/fmicb.2014.00481

18. Castillo H, Smith GB. Below-background ionizing radiation as an environmental cue for bacteria. Front Microbiol (2017) 8:177. doi:10.3389/fmicb.2017.00177

19. Morciano P, Cipressa F, Porrazzo A, Esposito G, Tabocchini MA, Cenci G. Fruit flies provide new insights in low-radiation background biology at the INFN LNGS. Radiat Res (2018) 3:217. doi:10.1667/RR15083.1

Conflict of Interest: The author declares that the research was conducted in the absence of any commercial or financial relationships that could be construed as a potential conflict of interest.

Copyright () 2021 Ianni. This is an open-access article distributed under the terms of the Creative Commons Attribution License (CC BY). The use, distribution or reproduction in other forums is permitted, provided the original author(s) and the copyright owner(s) are credited and that the original publication in this journal is cited, in accordance with accepted academic practice. No use, distribution or reproduction is permitted which does not comply with these terms. 